\title{
KNOWLEDGE OF PROSPECTIVELY RELEVANT INFORMATION IMPROVES SEARCH PERFORMANCE
}

\author{
Margit Höfler $^{1,2}$, Vanessa Kuwal ${ }^{1}$, Sebastian A. Bauch ${ }^{1}$, \& Anja Ischebeck ${ }^{1}$ \\ ${ }^{1}$ Institute of Psychology, University of Graz (Austria) \\ ${ }^{2}$ Department for Clinical Neurosciences and Preventive Medicine, Center for Dementia Studies, Danube, \\ University Krems (Austria)
}

\begin{abstract}
When searching for a target among distractors, we are able to focus our attention to those properties of the target or parts of the display relevant for the current search while ignoring irrelevant properties. However, it is unclear whether we can make use of irrelevant information when we are aware that these properties will become relevant in future searches. The aim of the current experiment was therefore to investigate whether and how the pre-knowledge about which parts of the display will become relevant in future searches affects the current and future searches in the same display. To this end, we had 20 participants perform two blocks of searches while their eye movements were recorded. During the first block, participants searched in 10 different search displays for different target letters that were superimposed on drawings of objects. Each search display was repeated 10 times, thus resulting in 100 searches per block. Half of the participants were instructed to ignore the objects accompanying the letters; the other half of the participants were told that, in a second block of searches, the objects would have to be searched for. Hence, in the latter case, participants were aware that the objects would become relevant in a further search task. The results showed that, in the first block (letter search), participants performed the search task equally fast, regardless of their pre-knowledge about the objects' prospective relevance in the second block. In the second block (object search), search performance was better for those participants who knew about the objects' relevance in advance compared to participants who had been told to ignore the objects. This suggests that participants are able to use relevant information from a previous search for future searches, without compromising search performance in the first search.
\end{abstract}

Keywords: Visual search, eye tracking, item relevance.

\section{Introduction}

When searching for a cup of strawberry yogurt of a familiar brand in the supermarket, you will perhaps restrict your attention to properties of the target objects that will help you find these objects quickly, for example, a brand's typical logo or color. At the same time you may ignore other properties of the target that are irrelevant to the search, for example, the precise shape of the cup. However, it is unclear whether we really succeed in using irrelevant information from previous searches in a later search environment, where this information eventually turns relevant. In order to preserve our limited working memory resources (Cowan, 2001), it would be efficient to process only such properties of targets that are relevant for the search task. There is evidence that memory is better for properties of targets objects that are relevant for the actual (visual search) task than for properties of target objects that are irrelevant (e.g., Williams, Henderson, \& Zacks, 2005). However, some irrelevant information might nevertheless be incidentally processed when a scene is inspected regardless of whether it will be of use in the future or not. For example, using a real-world setting in which participants were free to move around the environment while their eye movements were being recorded, Tatler and Tatler (2013) investigated memory for different objects by comparing the effect of three different task instructions. Participants were either asked to memorize as much as possible about all objects (undirected memory task) or only certain objects (directed memory task) in the environment, or received no memory instruction at all (free-viewing task). During a subsequent memory test, memory for four object properties (identity, color, location, and the relative distance to other objects) was tested. The results showed that memory performance was better in the directed and undirected memory task than in the free viewing task, with no differences between both (directed and undirected) memory tasks. However, object memory was above chance also in the free-viewing task. This indicated incidental memory for objects even if the task did not require 
memorization (see also Williams et al., 2005). This suggests that, although participants were able to restrict their attentional and memory resources to objects and properties that were essential for a certain task, task-irrelevant information also left some traces in memory.

It is unclear, however, whether task-irrelevant information is stored during visual serial search when participants do not expect a further search in the same environment. Võ and Wolfe (2012) had participants search repeatedly through different scenes for different target objects. Before this main search task, however, they were asked to pre-search the scenes for letters superimposed on some of the objects. When participants searched for target letters they also viewed the (irrelevant) objects located under those letters. Võ and Wolfe were interested in whether the irrelevant information about object identity during the pre-search would enhance search performance in the subsequent object search task. However, performance in the subsequent object search task did not change due to the pre-search (but see Hollingworth, 2012). The authors concluded that looking for objects (i.e. searching for specific objects) is different from looking at objects. Critically, however, participants in Võ and Wolfe were not aware that the objects behind those letters might become relevant in a future search. It is therefore possible that a more explicit instruction to memorize the objects might have enhanced search performance in the main search. In the present study, we investigated whether the knowledge about a future search modulated the processing of irrelevant item identity information in an earlier search. We assumed that memory for irrelevant item identity information would depend on the instruction. We either instructed participants that they could use the information in a subsequent search or not. To this end, we modified the repeated-search paradigm of Võ and Wolfe (2012). Participants had to complete two blocks of searches in which they searched displays repeatedly that consisted of ten objects superimposed by letters. All participants searched for letters superimposed on objects in the first block and for the objects in the second block. Half of the participants was explicitly told that they would have to search the display for the objects in the second block (object-relevant condition), whereas the other half was instructed to ignore the objects (object-irrelevant condition). If participants could use the irrelevant information during the first block as instructed, search performance in the second block should be faster in the object-relevant as compared to the object-irrelevant group.

\section{Methods}

\subsection{Design}

To test the effect of instruction on memory in repeated visual search we applied a 2 (search block: block 1, block 2) $\times 2$ (instruction type: object-relevant, object-irrelevant) design. During each block, they were presented with 10 different search displays that they had to search consecutively for 10 times each. In each search display, 10 items were presented. Items consisted of the picture of an object with a superimposed letter, such that object and letter were clearly discernible. All participants searched the displays for different target letters in the first block and for different target objects in the second block. Half of the participants were instructed that the objects behind the letters would become relevant during a further block (object-relevant condition) whereas the other half was instructed to ignore the objects (object-irrelevant condition). In each block, we measured manual response times and number of fixations needed to find the letters and objects.

\subsection{Participants}

20 participants (13 female, 7 male; average age: 24.0 years, range: $20-30$ years, $S D=3.4$ ) took part in the experiment. All participants reported normal or corrected to normal vision. The experiment was approved by the local ethics committee. Written informed consent was obtained from all participants.

\subsection{Stimuli}

100 different objects (individual size: $\max 1.8^{\circ}$ ) were chosen from the Microsoft® Clipart database and covered ten different categories (kitchen, bathroom, nature, animals, fairy-tale/fantasy characters, office, means of transportation, musical instruments, fruits, and sports equipment). In each of the ten displays, only items from the same category were presented. Stimuli were presented using Experiment Builder (SR Research, Canada) on a 21" CRT Monitor with a resolution of $1152 \times 864$ pixel and a refresh rate of $60 \mathrm{~Hz}$. Stimuli were presented randomly within an invisible $6 \times 6$ grid and could deviate from the center of each grid cell $\pm 0.16^{\circ}$. Each object was superimposed by a red letter in Arial font (bold). The letters were $0.45^{\circ}$ (20px) high and presented in red color (RGB: 255, 0, 0). They were randomly chosen from all capital letters of the Latin alphabet excluding the letter "I". We created the stimulus materials such that the letters did not occlude the objects completely and that the letters themselves were discriminable from the respective object (e.g., we did not use objects that were mainly of red color). The target letter or the name of the target object (high: $40 \mathrm{px} 0.91^{\circ}$ ) was presented in in red (RGB: $255,0,0)$ Arial font (bold). 


\subsection{Apparatus}

We used an EyeLink 1000 (SR Research, Canada) to track the eye movements of the participants. We tracked from one eye with an average spatial resolution better than $0.4^{\circ}$. Manual responses were measured using a Gamepad (Microsoft ${ }^{\circledR}$ Sidewinder). The distance from the participants’ eyes to the monitor was approximately $83 \mathrm{~cm}$.

\subsection{Procedure}

Participants sat in a dimly lit, sound proof cabin. They were instructed that they would have to search several search displays for different target letters. Participants were instructed to search for the letters in Block 1 and either ignore the objects behind the letters (objects-irrelevant condition) or to process these objects as they would become relevant in a later block of searches (objects-relevant condition).

Each block started with a 9-point calibration of the eye tracker. In both blocks, each trial started with the presentation of a fixation disc (which also served as a drift correction for the eye tracker) at the center of the screen. Immediately afterwards, the target letter (in the first block) or the target object (in the second block) was presented for 1,000 msec (see Figure 1). The target object was written in German. Then the search display was presented. The task of the participants was to search for the respective target in the search display and to press the trigger on the game pad with the right index finger when the target was found. The target was always present. Participants were told to search as fast and as accurately as possible. With the button press, a new trial started with the presentation of a new target. In the first block, participants searched the same display for ten different target letters before a new display was presented. In the second block, we presented search displays with the objects only and participants were asked to search for a target object. As the overlying letters were omitted in the displays, participants could not rely on possibly learned association between the letters and objects when they searched for the letters in the first search block. Within the 10 searches in the same display, the same target (letter or object) was never searched twice. The order of the presentation of the displays was randomized as well as the order of the targets. There was a short pause between the first and the second block. The participants completed 10 practice trials with a different set of items than in the actual experiment. The experiment lasted about 45 minutes.

Figure 1. Sequence of events in Block 1 and 2. Each of the ten different displays was presented ten times consecutively. In Block 1, participants had to search for different target letters in displays with ten objects with the target letters superimposed on them. Half of the participants were instructed to ignore the objects behind the letters, the other half to process them. In Block 2, participants searched for different target objects (letters removed).

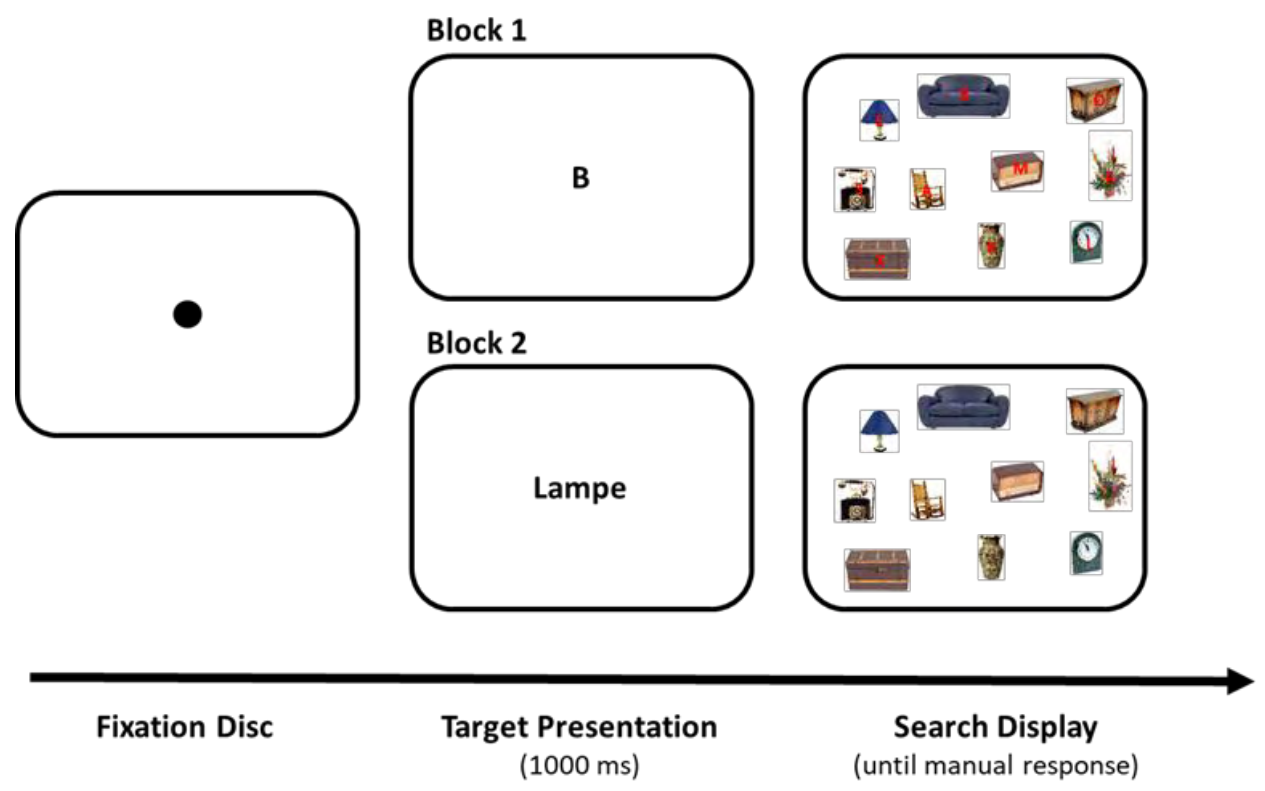




\section{Results and discussion}

In total, 4,000 trials were available for analysis (20 participants $\mathrm{x} 200$ trials). For each fixation, we computed the Euclidean distances relative to all items in the display and the item with the smallest distance was then determined as the item fixated. We excluded all trials in which participants did not fixate the respective target item during the trial. This was the case for 504 trials $(12.6 \%)$ of the trials.

We computed the mean response time, averaged across individual means separately for each block and instruction group (see Figure 2). In Block 1, participants needed on average 2,050 ms $(S D=225)$ to find the target letter in the object-relevant condition and $1,939 \mathrm{~ms}(S D=283)$ in the object-irrelevant condition. In Block 2, participants needed 1,056 ms $(S D=233)$ if they had been instructed to process the objects in Block 1 and $1,190 \mathrm{~ms}(S D=225)$ if they had been instructed to ignore the objects. A $2 \times 2$ ANOVA with block as within and instruction (object relevant vs. object irrelevant) as between-factor revealed a significant main effect of block, $F(1,18)=294.96, p<.001, \eta_{p}{ }^{2}=0.93$, but no main effect of instruction, $F<1$. However, the interaction was significant, $F(1,18)=5.03, p=.038$, $\eta_{p}{ }^{2}=0.22$. In particular, the difference in search performance between Block 1 and Block 2 was reliably larger for the object-relevant group (994 ms) compared to the object-irrelevant group (749 ms), $t(18)=2.43, p=.038$. This suggests that participants who were instructed to process the objects during Block 1 benefited in Block 2 from this information more than participants who were instructed to ignore the objects.

To investigate whether this benefit in the second block is also reflected in the oculomotor system, we conducted a further ANOVA with the number of fixations until the first target fixation as dependent variable. Participants inspected in the first block $9.3(S D=0.77)$ items in the object-relevant condition and $8.7(S D=1.17)$ item in the object-irrelevant condition. In the second block they fixated 6.3 items both in the object-relevant and irrelevant condition ( $S D=0.8$ and 1.3 , respectively). We found a significant main effect of block with fewer fixations in the second block than in the first block, $F(1,18)=99.80, p<.001, \eta_{p}{ }^{2}=0.85$. The main effect of instruction as well as the interaction was not significant, both $F<1$. This suggests that number or fixated items during search is less sensitive for measuring the effects of task instruction. Further research is necessary to investigate how participants benefit from pre-knowledge about prospective item relevance.

Figure 2. Mean response time separately for each block and instruction condition.

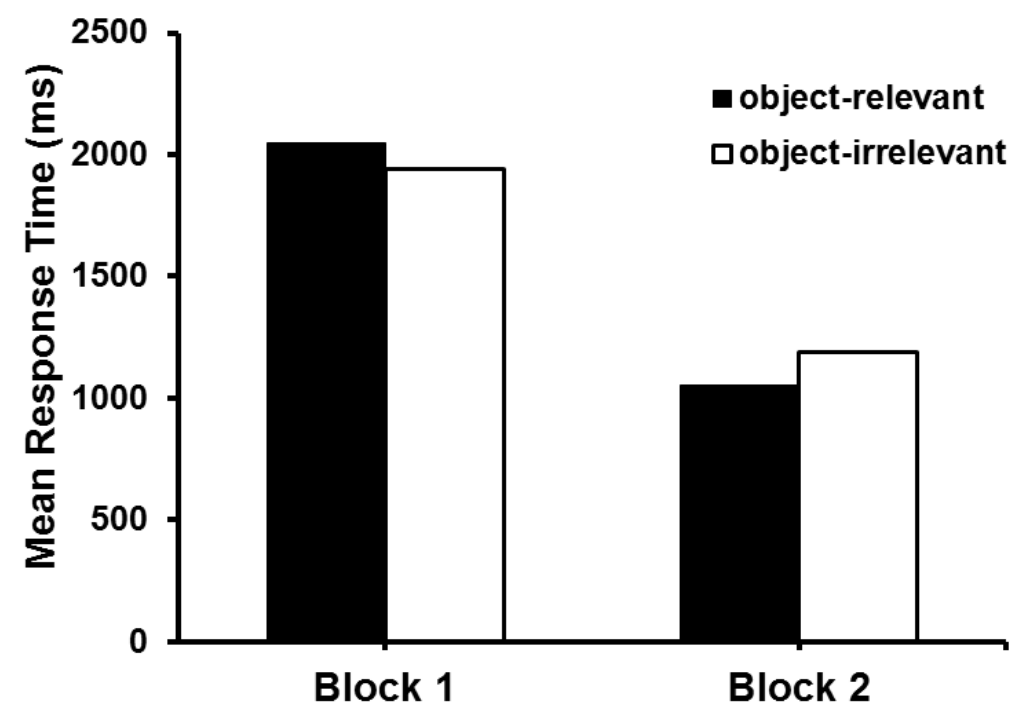

\section{Conclusion}

In the present paper we investigated whether knowledge about the future relevance of currently irrelevant target properties modulated search times in current as well as in future searches. We found that participants that were explicitly informed about the future relevance of the currently irrelevant target property (i.e. the object identity) showed a greater difference in search times between search block one and two. Our findings show, at least when analyzing the manual responses, that participants could benefit from irrelevant information during visual search when they were informed that this information would become relevant in a later search. This indicates that traces of irrelevant information can be kept in memory during visual search. 


\section{Acknowledgements}

This work was supported by a grant from the Austrian Science Fund (FWF): P 28546 to M.H. We are grateful to Jasmin Jennetten for her support during the preparation of the manuscript.

\section{References}

Hollingworth, A. (2012). Task Specificity and the Influence of Memory on Visual Search: Comment on Höfler, M., Gilchrist, I.D., \& Körner, C. (2015). Guidance toward and away from distractors in repeated visual search. Journal of Vision, 15(5), 1-14. doi:10.1167/15.5.12

Oberauer, K. \& Eichinger, S. (2013). Visual working memory declines when more features must be remembered for each object. Memory \& Cognition, 41, 1212 - 1227.

Tatler, B. \& Tatler, S. L. (2013). The influence of instructions on object memory in a real-world setting. Journal of Vision, 13(2):5. doi: 10.1167/13.2.5.

Võ, M. L.-H., \& Wolfe, J. M. (2012). When does repeated search in scenes involve memory? Looking at versus looking for objects in scenes. Journal of Experimental Psychology: Human Perception and Performance, 38(1), 23-41.

Williams, C.C., Henderson, J.M., \& Zacks, R. T. (2005). Incidental visual memory for targets and distractors in visual search. Perception \& Psychophysics, 67(5) 816-827. https://doi.org/10.3758/BF03193535 